\title{
Perte de rendement due aux maladies cryptogamiques sur blé tendre d'hiver. Construction et validation d'un modèle de l'effet du système de culture
}

\author{
C Chevalier-Gérard ${ }^{1 *}$, JB Denis ${ }^{2}$, JM Meynard $^{3}$ \\ 1 INA-PG, laboratoire d'agronomie, 16, rue Claude-Bernard, F75231 Paris cedex 05; \\ 2 INRA, laboratoire de biométrie, route de Saint-Cyr, F78026 Versailles; \\ 3 INRA, station d'agronomie, F78850 Thiverval-Grignon, France
}

(Reçu le 18 mai 1993 ; accepté le 11 mai 1994)

\begin{abstract}
Résumé - Avec l'objectif de concevoir, pour le blé tendre d'hiver, des itinéraires techniques à bas niveau d'intrants, nous avons élaboré un modèle linéaire simple d'estimation de la perte de rendement due aux maladies d'origine cryptogamique, en l'absence de traitement fongicide. Les effets d'éléments du système de culture et de l'année climatique sur les dégâts dus aux maladies sont quantifiés. Nous présentons ici le modèle élaboré à partir de 454 essais fongicide des régions Haute-Normandie et Picardie, de 1978 à 1991, sa validation et son intérêt pour la conception d'itinéraires techniques à bas niveau d'intrants fongicide.
\end{abstract}

blé d'hiver / maladies cryptogamiques / prédiction de perte de rendement / système de culture / modèle linéaire

Summary - Estimation of yield loss caused by fungal diseases on winter wheat: construction and validation of a model taking into account the effects of the cropping system. The risk of yield loss caused by fungal diseases on winter wheat has always been believed to be a limiting factor that farmers could only eliminate by using fungicides. These chemicals now represent about $25 \%$ of the supply costs in France. In order to grow winter wheat with low fungicidal inputs we must take into account possibilities other than fungicides to reduce the damage caused by disease. $A$ statistical linear model of prediction that estimates the yield loss caused by fungal diseases has been built. The important variable is the percentage of the yield obtained without disease. The effects of the climate and various elements of the cropping system on yield loss were quantified: previous crop type; variety; and planting date. In this paper, we develop a model obtained from 454 fungicide trials in Haute-Normandie and Picardie (in northern France) from 1978 to 1991. The various steps of the conception of the model of prediction are discussed. Its validation is discussed together with its importance for the construction of new methods of growing wheat with low fungicide inputs.

winter wheat / fungal diseases / yield-loss prediction / crop management / linear model

\footnotetext{
* Correspondance et tirés à part
} 


\section{INTRODUCTION}

Les dernières décennies ont vu une forte intensification de la culture du blé, cohérente avec la politique de soutien des prix menée par la Communauté économique européenne. Le changement de politique agricole se traduit par une forte baisse du prix du blé qui impose la conception de nouveaux itinéraires techniques à niveaux d'intrants plus faibles. Cet ajustement de la conduite de la culture à de nouveaux rapports de prix ne pourra être réalisé que si l'on dispose de modèles prédictifs de l'effet des techniques culturales sur le rendement (Meynard, 1991). Cet article s'inscrit dans un objectif de réalisation d'un tel modèle : le logiciel Déciblé (Aubry et al, 1992 ; organismes impliqués : Institut national de la recherche agronomique, Institut technique des céréales et des fourrages et Institut national agronomique de Paris-Grignon), conçu pour l'aide à la décision stratégique. L'objectif est de comparer différents "plans d'action" (ensemble de règles de décision) pour l'itinéraire technique, quant à leurs résultats sur le rendement, la marge brute, l'azote non utilisé par la culture... Déciblé fonctionne sur des scénarios climatiques d'années passées. II permet d'estimer des fréquences d'occurrence de telle ou telle valeur de ses différentes variables de sortie ; il constitue un outil de choix des plans d'action les mieux adaptés à un cahier des charges donné.

En France, les fongicides constituent actuellement le premier poste de dépenses dans les charges d'approvisionnement (Meynard, 1991). II est donc indispensable qu'un outil d'aide à la décision stratégique, tel que Déciblé, prenne en compte les effets de l'ensemble du système de culture sur les dégâts liés aux maladies : il doit fournir, pour chaque système de culture, une fonction de distribution selon les années de la perte de rendement due aux maladies en l'absence de traitement fongicide.

Les différents modèles de maladies existant sont des modèles d'évolution des symptômes maladie par maladie (Rapilly, 1991) ; ils ne permettent pas d'estimer les dégâts dus à l'ensemble du complexe parasitaire. De plus, ils ne prennent en général pas en compte les effets du système de culture.

Ce travail vise à déterminer, à l'échelle de la parcelle, l'impact de différents éléments du système de culture sur les dégâts potentiels des maladies. II propose de répondre à cette question à travers une approche de modélisation de l'effet des éléments du système de culture et du climat sur cette perte de rendement. Nous ne cherchons pas à prédire une perte de rendement pour une nouvelle année climatique, mais à utiliser des essais d'années climatiques passées pour fournir une fréquence d'obtention de telle ou telle valeur de perte de rendement, pour une parcelle donnée.

\section{MATÉRIELS : LE RÉSEAU D’ESSAIS}

Pour modéliser la perte de rendement, il est nécessaire de disposer d'essais fongicide comportant une partie traitée de façon à éradiquer les maladies contre lesquelles il existe des matières actives efficaces, et une partie non traitée. Cette base de données doit inclure le nombre le plus important possible d'années climatiques et, pour chaque année, une gamme étendue de combinaisons de techniques culturales, afin de mettre en évidence non seulement les effets des différentes techniques et leurs interactions, mais aussi, les effets des interactions entre techniques et climat.

Le texte donne les résultats de l'étude portant sur une région regroupant Haute-Normandie et Picardie. Une collecte d'essais fongicide a été réalisée auprès de l'ITCF et des Chambres d'agriculture : après avoir éliminé 141 points d'obsenvations aux caractéristiques inconnues telles que type de sol (59\% de ces essais), quantité d'engrais apporté, précédent cultural, date de semis, lieu de l'essai, on dispose de 454 essais exploitables pour la modélisation. Ils s'inscrivent dans une période allant de 1978 à 1991, période de relative homogénéité de l'efficacité des produits fongicides.

Les rendements sont connus ainsi que certaines caractéristiques de la parcelle (lieu de l'essai, type, profondeur ou texture du sol) et du système de culture telles que : nature du précédent cultural ${ }^{\star}$, date de semis, variété, quantité d'engrais azoté apporté.

Le problème majeur de ce réseau réside dans le manque d'informations concernant certaines parcelles. Pour 14 essais, nous ne disposons que du nom vernaculaire du sol pour retrouver le type pédologique. Pour 81 essais, il a fallu estimer la classe de profondeur de sol à partir du type de sol.

Les renseignements sur le système de culture sont également très incomplets : seuls 213 essais ont une indication de densité de peuplement, et la nature de l'antéprécédent* n'est disponible que dans 108 cas.

La structure de notre jeu de données est fortement déséquilibrée. Parmi tous les départements de la région étudiée, le département de l'Aisne représente seulement $9 \%$ des essais. Ce réseau est caractérisé par une majorité de sols profonds (supérieure à $80 \mathrm{~cm}$ dans $85 \%$ des cas), de précédents autres que les céréales à paille ( $86 \%$ ) et de variétés sensibles au piétin-verse (95\% des notes de résistance au piétin-verse

\footnotetext{
* Précédent : culture antérieure à la culture étudiée ; antéprécédent : culture antérieure au précédent.
} 
sont comprises entre 1, «très sensible», et 4, "assez sensible»). Une analyse en composantes principales (ACP) pour les 454 observations et les 9 variables étudiées (notes de résistance à la rouille jaune, aux septorioses, au piétin-verse, à la fusariose de l'épi, à l'oïdium et à la rouille brune, quantité d'azote disponible, date de semis et distance au littoral) révèle que, en tendance ( 3 axes expliquent $55 \%$ de la variabilité de l'ensemble des données), plus le semis est précoce, plus la quantité d'azote disponible est grande. La fréquence des fortes quantités d'azote et des semis précoces s'accroît pour les années récentes, correspondant au sens général de l'évolution de ces techniques culturales dans la dernière décennie (Meynard, 1991).

\section{MÉTHODE : \\ RECHERCHE D'UN MODÈLE PRÉDICTIF}

\section{Principe de la modélisation}

En l'absence de données bibliographiques sur les interactions entre pathogènes, nous sommes obligés d'envisager globalement l'ensemble des pathogènes contre lesquels il existe des fongicides efficaces, que nous appellerons le «complexe parasitaire», c'est-àdire les agents du piétin-verse (Pseudocercosporella herpotrichoïdes Fron Deighton), de l'oïdium (Erysiphe graminis DC ex Mérat), de la rouille brune (Puccinia recondita Rob ex Desm), de la rouille jaune (Puccinia striiformis Westend), des septorioses (Septoria tritici Rob ex Desm et Septoria nodorum Rob ex Desm) et de la fusariose de l'épi (Fusarium roseum Berck, Fusarium culmorum Berk et Fusarium graminearum Berk).

Les dégâts consécutifs aux autres maladies cryptogamiques telles que le piétin-échaudage (Gaeumannomyces graminis (Sacc) van Arx et Olivier) ou le rhizoctone (Rhizoctonia cerealis van der Hoeven) sont très difficiles à évaluer, puisqu'on ne connaît pas de fongicides efficaces contre ces pathogènes telluriques.

Il existe très peu de connaissances sur la façon dont les techniques culturales jouent sur les symp- tômes, et les phénomènes complexes influençant la traduction des symptômes en dégâts. La perte de rendement est modélisée directement à partir des éléments des systèmes de culture, des climats et des sols, sans passer par une estimation des symptômes de maladies. Le modèle recherché doit être utilisable dans une grande gamme de situations induites par des systèmes de cultures et ne comporter qu'un petit nombre de paramètres. Nous ne pouvions pas, en conséquence, construire un modèle de type mécaniste. Le but est d'élaborer un modèle d'ajustement statistique aux données expliquant la perte de rendement, "variable de sortie", à l'aide des "variables d'entrée" que sont : itinéraire technique, histoire culturale, sol et climat. Le modèle le plus général envisagé est linéaire du type :

Perte de rendement due aux maladies = Effets principaux + Effets des interactions

Les différents termes de ce modèle sont explicités dans les paragraphes qui suivent.

\section{La variable à expliquer : la perte relative de rendement due aux maladies}

L'augmentation, avec le potentiel de rendement, du dégât absolu dû aux maladies est observée par de nombreux auteurs (Lechapt, 1983 ; Meynard, 1985 ; Stoop, 1992). Nos données corroborent cette relation (tableau I) : dans toutes les régions, une corrélation positive est observée entre perte de rendement (rendement traité - rendement non traité) et rendement traité. C'est pourquoi nous avons choisi de modéliser, en fonction du système de culture et du climat, la perte de rendement relative, exprimée en pourcentage:

$$
100 \text { (RDT traité - RDT non traité) / RDT traité }
$$

avec : RDT traité = Rendement supposé "sans maladie" ; RDT non traité = Rendement non traité avec des fongicides.

Le modèle statistique utilisé est le modèle linéaire général qui permet de mêler les effets de facteurs et de covariables ; les paragraphes suivants précisent quels effets ont été envisagés.

Tableau I. Coefficients de corrélation ( $r$ ) entre perte de rendement et rendement traité («sans maladie») dans différentes régions céréalières.

\section{Haute-Normandie et Picardie 454 observations}

\section{He-de-France et Beauce}

674 observations
Poitou-Charentes 409 observations

$$
r
$$

0,39

0,35 


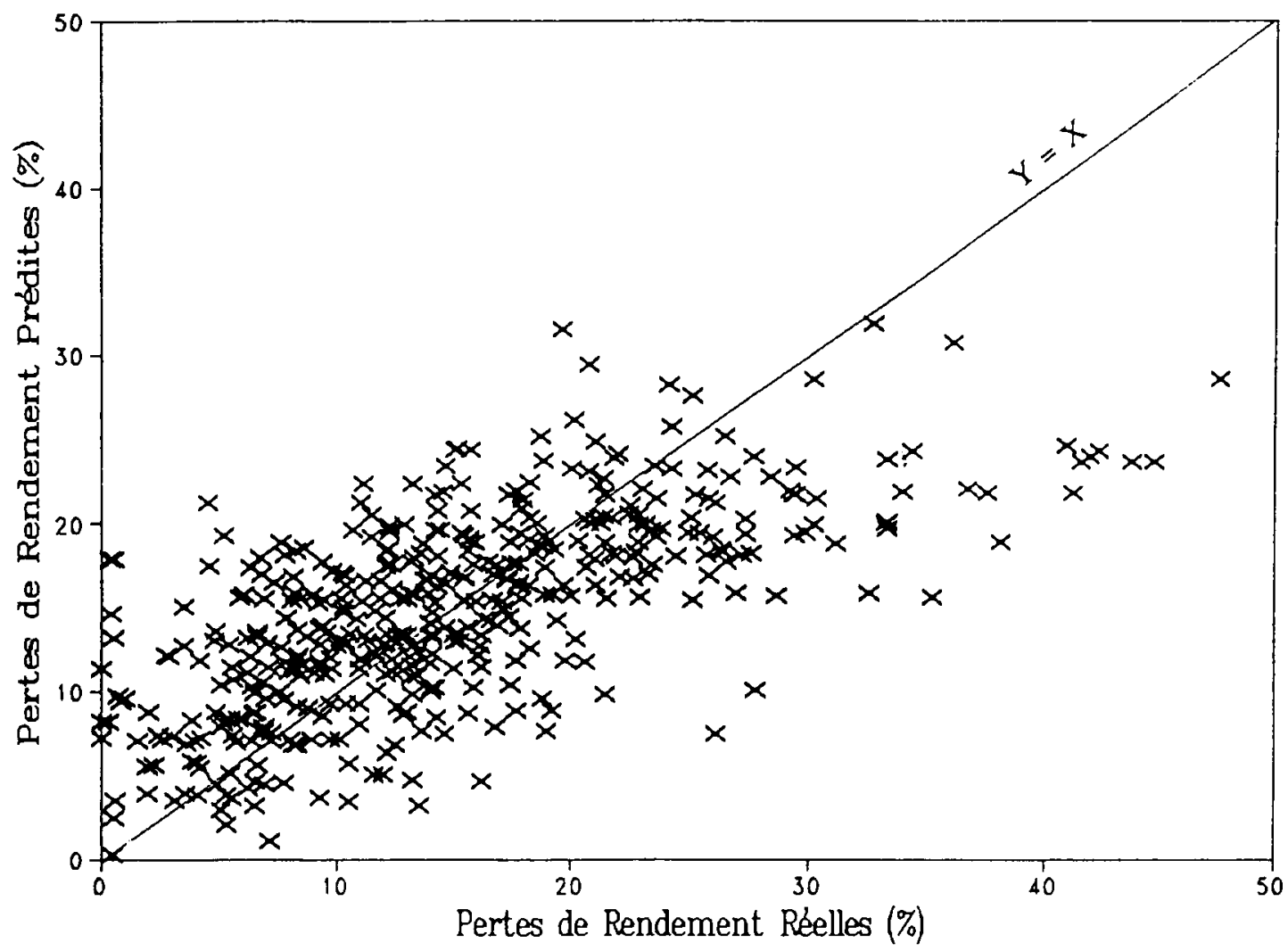

Fig 1. Pertes de rendement prédites en fonction des pertes de rendement réelles. Première bissectrice (454 observations).

\section{Les effets principaux}

Dans la suite du texte, les termes de "covariable» et de «facteur» seront employés pour désigner respectivement une variable quantitative, et une variable qualitative servant à expliquer la perte de rendement relative.

À l'aide de travaux bibliographiques, l'identification des covariables et des facteurs importants parmi les caractéristiques du climat, du sol, et ceux des éléments du système de culture qui ont un effet sur les dégâts dus aux maladies, est recherchée. Une priorité des facteurs et covariables retenus a ensuite été établie afin de limiter le nombre de covariables et de facteurs explicatifs, car prendre en compte trop de paramètres diminue la valeur prédictive du modèle.

Les gammes de variation des facteurs et des covariables sont données dans le tableau II.

\section{Le climat}

L'effet du climat a été pris en compte non pas par des variables climatiques analytiques, mais par un facteur "année" non ordonné, avec 13 degrés de liberté, et le facteur "distance à la mer», comportant 3 classes : 0-20, 20-40 et $>40 \mathrm{~km}$, correspondant à des zones de températures et de précipitations différentes.

\section{L'histoire culturale}

Les agents du piétin-verse et des septorioses survivent sur les pailles des céréales (Steinbrenner et Seidel, 1982 ; Cook et Veseth, 1991 ; Polley et Thomas, 1991). Dans notre modèle, le facteur «nature du précédent cultural» est inclus et comprend 2 modalités : "céréales à paille" et "autres précédents". L'effet de l'antéprécédent est étudié seulement sur les résidus du modèle pour les 108 essais pour lesquels la nature de l'antéprécédent est connue.

\section{La variété}

La variété elle-même joue un rôle important vis-à-vis des maladies (Huet, 1983a ; Cook et Veseth, 1991). Dans le modèle, les variétés sont caractérisées par leurs 6 notes de résistance aux maladies du «complexe parasitaire". Ces notes sont issues des bulletins du GEVES (Groupement d'étude et de contrôle des variétés et des semences) (INRA-GEVES, 1980, 1986, 1991 et 1992) et de l'ITCF $(1977,1980,1986$ et 1991) et sont considérées comme des covariables.

\section{La densité de semis}

Une forte densité de semis favorise les maladies, et notamment le piétin-verse (Huet, 1983b, 1986b) mais 
Tableau II. Domaines de variation des facteurs et covariables explicatives pour les régions Haute-Normandie et Picardie.

\begin{tabular}{|c|c|c|c|c|c|c|}
\hline $\begin{array}{l}\text { Facteurs } \\
\text { et covariables }\end{array}$ & Nature & $\begin{array}{c}\text { Unité } \\
\text { de mesure }\end{array}$ & Moyenne & Minimum & Maximum & $\begin{array}{l}\text { Ecart } \\
\text { type }\end{array}$ \\
\hline Année & $\begin{array}{c}\text { Facteur } \\
14 \text { modalités }\end{array}$ & - & - & 1978 & 1991 & - \\
\hline Distance à la mer & $\begin{array}{l}\text { Facteur } \\
3 \text { modalités }\end{array}$ & $\mathrm{km}$ & - & $0-20 \mathrm{~km}$ & $>40 \mathrm{~km}$ & - \\
\hline $\begin{array}{l}\text { Note de résistance } \\
\text { à la rouille jaune }\end{array}$ & Covariable & $\begin{array}{l}\text { Note de } \\
1 \text { à } 9\end{array}$ & 4,8 & 1 & 8 & 2,4 \\
\hline $\begin{array}{l}\text { Note de résistance } \\
\text { aux septorioses }\end{array}$ & Covariable & $\begin{array}{l}\text { Note de } \\
1 \text { à } 9\end{array}$ & 3,6 & 1,5 & 6 & 1,2 \\
\hline $\begin{array}{l}\text { Note de résistance } \\
\text { au piétin-verse }\end{array}$ & Covariable & $\begin{array}{l}\text { Note de } \\
1 \text { à } 9\end{array}$ & 2,8 & 1 & 6 & 1,0 \\
\hline $\begin{array}{l}\text { Note de résistance } \\
\text { à la fusariose } \\
\text { de l'épi }\end{array}$ & Covariable & $\begin{array}{l}\text { Note de } \\
1 \text { à } 9\end{array}$ & 4,9 & 2 & 7 & 1,4 \\
\hline $\begin{array}{l}\text { Note de résistance } \\
\text { à l'oïdium }\end{array}$ & Covariable & $\begin{array}{l}\text { Note de } \\
1 \text { à } 9\end{array}$ & 4,6 & 1 & 8 & 1,4 \\
\hline $\begin{array}{l}\text { Note de résistance } \\
\text { à la rouille brune }\end{array}$ & Covariable & $\begin{array}{l}\text { Note de } \\
1 \text { à } 9\end{array}$ & 3,7 & 1 & 8 & 2,3 \\
\hline Nature du précédent & $\begin{array}{c}\text { Facteur } \\
2 \text { modalités }\end{array}$ & - & - & $\begin{array}{l}\text { Céréale } \\
\text { à paille }\end{array}$ & Autre & - \\
\hline Date de semis & Covariable & $\begin{array}{c}{ }^{\circ} \mathrm{C} \text { jour } \\
\text { en base } 0\end{array}$ & 536,8 & 0 & 896 & 149,4 \\
\hline $\begin{array}{l}\text { Quantité d'azote } \\
\text { disponible }\end{array}$ & Covariable & $\mathrm{kg} / \mathrm{ha}$ & 265,8 & 117 & 355 & 48,7 \\
\hline
\end{tabular}

cette covariable n'a pu être intégrée dans le modèle, n'étant pas disponible dans de très nombreux essais. L'effet de la densité de la population végétale est étudié seulement sur les résidus du modèle pour les quelques essais pour lesquels la densité est connue.

\section{La date de semis}

Tous les auteurs s'accordent pour dire qu'un semis précoce augmente la gravité du piétin-verse (Hollins et Scott, 1980 ; Rapilly, 1982 ; Steinbrenner et Hoflich, 1984 ; Huet, 1986a ; Schultz et al, 1990) et, dans une moindre mesure, les maladies du feuillage (Palti, 1981 ; Caron, 1988 ; Polley et Thomas, 1991). La majorité des contaminations hivernales en souches normales de Pseudocercosporella herpotrichoïdes est réalisée au 31 janvier (Rapilly et al, 1979). L'effet, situation du cycle cultural par rapport aux périodes climatiques favorables au champignon, est traduit, dans le modèle, par la somme de températures (en base $0^{\circ} \mathrm{C}$ ) depuis le semis jusqu'au 31 janvier. Cette covariable rend également compte de l'effet de la température en elle-même sur la relation hôte-pathogène.

\section{Le statut de nutrition azotée de la population végétale}

L'azote intervient sur les effets des pathogènes, notamment les rouilles (Palti, 1981), les septorioses (Oppitz et Hoeser, 1978), l'oïdium (Caron, 1988) et le piétin-verse (Steinbrenner et Höflich, 1984), par l'effet de la densité et de la turgescence de la population végétale, de même que sur le niveau de sensibilité de la plante aux maladies (Palti, 1981 ; Chaboussou, 1985). En l'absence de données sur l'azote absorbé par la plante, on retient, pour notre modèle, une covariable "azote disponible" calculée d'après la méthode du bilan (Hébert, 1969 ; Rémy et Hébert, 1977). 


\section{Les interactions}

Les combinaisons des différents facteurs et covariables ne sont pas toutes présentes dans notre jeu de données. Ceci amène à limiter le nombre d'interactions considérées a priori dans le modèle. Ainsi, le précédent cultural étant toujours un précédent «non céréale à paille" pour 6 des années, nous ne pouvons pas prédire correctement l'interaction Année $x$ Précédent. En revanche, l'étude des relations entre "année et notes de résistance aux maladies", et entre "année et date de semis" ne révèle pas de corrélation importante entre ces critères.

Deux types d'interactions ont été sélectionnés pour le rôle important qu'elles jouent sur les dégâts : Année $x$ Note de résistance à chacune des maladies du "complexe parasitaire" (6 interactions) et Année $x$ Date de semis. Chacune de ces interactions comporte 13 degrés de liberté (tableau II).

\section{Le modèle linéaire}

II prend en compte les effets principaux des différents covariables et facteurs retenus ainsi que leurs interactions (tableau III).

Des régressions linéaires multiples descendantes sont effectuées afin de déterminer les covariables et les facteurs importants et quantifier les effets de ces critères. La procédure GLM (General Linear Model) du logiciel de traitement statistique SAS (Statistical
Analysis System, SAS Institute Inc, 1989) est utilisée. La démarche est la suivante. À partir du modèle complet, qui est supposé "vrai» d'après les travaux bibliographiques, des covariables et des facteurs sont progressivement éliminés en fonction des valeurs des probabilités associées aux statistiques de test des hypothèses nulles : "L'apport de la covariable (ou du facteur) dans le modèle n'est pas significatif.» On élimine la covariable (ou le facteur) si la probabilité correspondante est supérieure à 0,10 . Le processus se poursuit jusqu'à ce que tous les facteurs et les covariables soient significatifs au niveau $10 \%$ (risque de première espèce de rejeter l'hypothèse nulle alors qu'elle est vraie) permettant de retenir le modèle correspondant. L'étude de la distribution des résidus de ce modèle, en fonction des différents facteurs et covariables explicatifs, permet de s'assurer qu'aucun effet systématique, par exemple quadratique, n'est oublié.

$\mathrm{Si}$ les pertes. de rendement estimées sont aberrantes (au-delà de 100\%) pour certaines combinaisons des variables d'entrée existant dans la pratique agricole et non représentées dans l'échantillon des données, le modèle est simplifié, en retirant en premier lieu les interactions : le modèle obtenu, après ces simplifications, est qualifié de "prédictif».

\section{La validation}

Elle consiste à juger la qualité de la prédiction fournie par le modèle prédictif et est réalisée de 3 manières complémentaires :

Tableau III. Modèle le plus général, pour la Haute-Normandie et la Picardie : covariables et facteurs pris en compte.

100 (RDT traité - RDT non traité)

\begin{tabular}{|c|c|c|}
\hline RDT traité & $\begin{array}{l}\text { + Année } \\
\text { + Distance à la mer } \\
\text { + Note de résistance à la rouille jaune } \\
\text { + Note de résistance aux septorioses } \\
\text { + Note de résistance au piétin-verse } \\
\text { + Note de résistance à la fusariose de l'épi } \\
\text { + Note de résistance à l'oïdium } \\
\text { + Note de résistance à la rouille brune } \\
\text { + Nature du précédent } \\
\text { + Date de semis } \\
\text { + Azote disponible } \\
\text { + Année } \times \text { Note de résistance à la rouille jaune } \\
+ \text { Année } \times \text { Note de résistance aux septorioses } \\
\text { + Année } \times \text { Note de résistance au piétin-verse } \\
+ \text { Année } \times \text { Note de résistance à la fusariose de l'épi } \\
+ \text { Année } \times \text { Note de résistance à l'oïdium } \\
+ \text { Année } \times \text { Note de résistance à la rouille brune } \\
+ \text { Année } \times \text { Date de semis }\end{array}$ & $\begin{array}{r}13 \\
2 \\
1 \\
1 \\
1 \\
1 \\
1 \\
1 \\
1 \\
1 \\
1 \\
13 \\
13 \\
13 \\
13 \\
13 \\
13 \\
13\end{array}$ \\
\hline
\end{tabular}


i) Vérifier la cohérence du modèle avec la bibliographie. Des analyses de sensibilité sont effectuées : faire varier la valeur d'une covariable (ou d'un facteur) d'entrée du modèle et étudier les modifications induites sur la perte de rendement prédite.

ii) Étudier l'histogramme des résidus du modèle et tracer la représentation cartésienne des valeurs prédites et des valeurs observées.

iii) Apprécier la qualité du modèle pour prédire des pertes de rendement, à partir de 2 méthodes :

- sous l'hypothèse que le modèle est sans biais : la qualité prédictive d'un modèle. Elle est jugée par l'estimation de l'erreur quadratique moyenne sur la structure particulière des données (espérance mathématique de la somme de carrés des différences entre les observations et le modèle proposé ; Bunke and Droge, 1984 ; Wallach and Goffinet, 1987), MSE pour la variable étudiée (d'après le Mean Squared Error Predictor, Wallach, 1991).

Son expression analytique pour le modèle prédictif est la suivante :

$$
\mathrm{MSE}=[1+(p / n)] \hat{\sigma}^{2}
$$

avec : $n:$ nombre total d'observations ; $p:$ nombre de paramètres du modèle prédictif ; $\hat{\sigma}^{2}$ : variance résiduelle pour le modèle prédictif.

Le modèle prédictif est comparé à un sous-modèle simple, où les valeurs prédites sont égales aux moyennes annuelles de pertes relatives de rendement observées. Le meilleur modèle pour la prédiction est celui qui le MŜ́ le plus faible.

- sans supposer que le modèle est sans biais : la validation croisée. Au sens d'une "leave-one-out Cross Validation" (Linhart et Zucchini, 1986), elle consiste à retirer chaque fois une observation parmi les $n$ observations de la population de données, estimer les paramètres du modèle sur les $n-1$ observations restantes, et utiliser ces estimations pour calculer la perte de rendement prédite pour l'observation retirée. Cette opération, effectuée $n$ fois, donne $n$ valeurs prédites. La qualité des prédictions est estimée par l'erreur quadratique moyenne pour la validation croisée, MSEcv (Wallach, 1991):

$$
M \hat{S E}_{\mathrm{CV}}=(1 / n) \sum_{k=1}^{n}\left(Y_{k}-\hat{Y}_{k} c v\right)^{2}
$$

avec : $Y_{k}$ : valeur de perte ge rendement relative observée poue le $k^{e}$ individu; $\gamma_{k} c v$ : valeur de perte de rendement relative prédite par la méthode de la validationc croisée, pour la $k^{e}$ observation ; $n$ : nombre total d'observations.

\section{RÉSULTATS. APPLICATION À LA RÉGION HAUTE-NORMANDIE ET PICARDIE}

Le modèle retenu uniquement à partir des tests significatifs, pour les régions Haute-Normandie et Picardie, est décrit dans le tableau IV. Son coefficient de détermination $\left(R^{2}\right)$ est de $56 \%$. Les effets de ses facteurs et de ses covariables, la date de semis mise à part, sont hautement significatifs : les probabilités associées aux tests des hypothèses nulles - «l'apport du critère n'est pas significatif» - sont comprises entre 0,000 1 et 0,0095 . En revanche, la date de semis n'est pas significative ; cette covariable est tout de même conservée, car son interaction avec l'année est très significative ; les paramètres des interactions correspondent à des corrections par rapport aux effets principaux.

Tableau IV. Modèles Haute-Normandie et Picardie : premier modèle retenu a et modèle prédictif $b$. Valeurs de probabilités limites associées à chacun des termes.

Perte de rendement relative

Covariables et facteurs

Proba $>F$ a Proba $>F^{\star}, \mathrm{b}$

100 (RDT traité - RDT non traité)

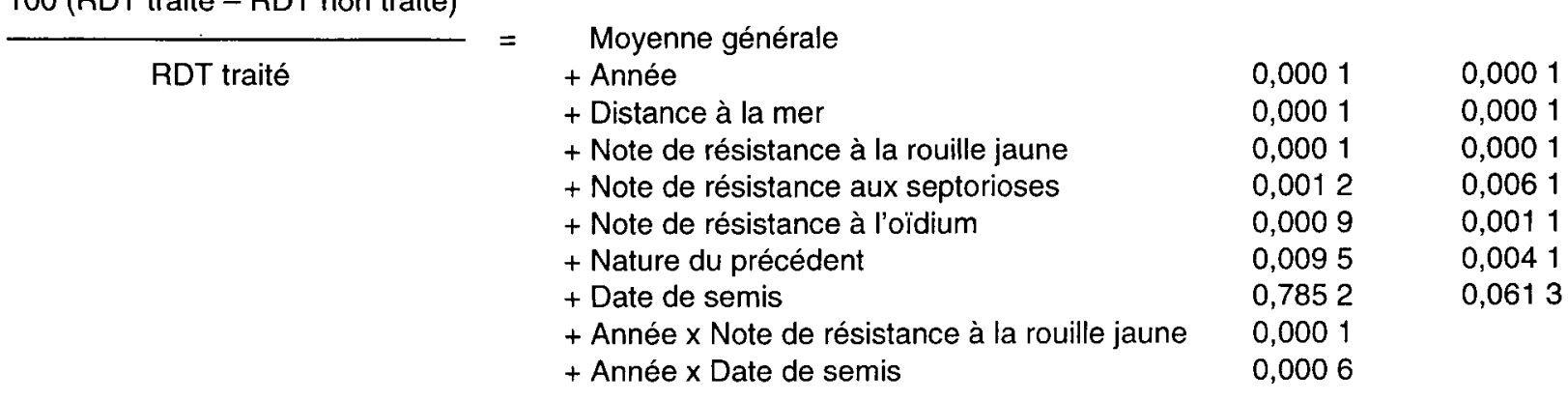

\footnotetext{
* Probabilités limites associées aux tests des hypothèses nulles; a pour le premier modèle retenu; ${ }^{b}$ pour le modèle prédictif (sans interactions).
} 


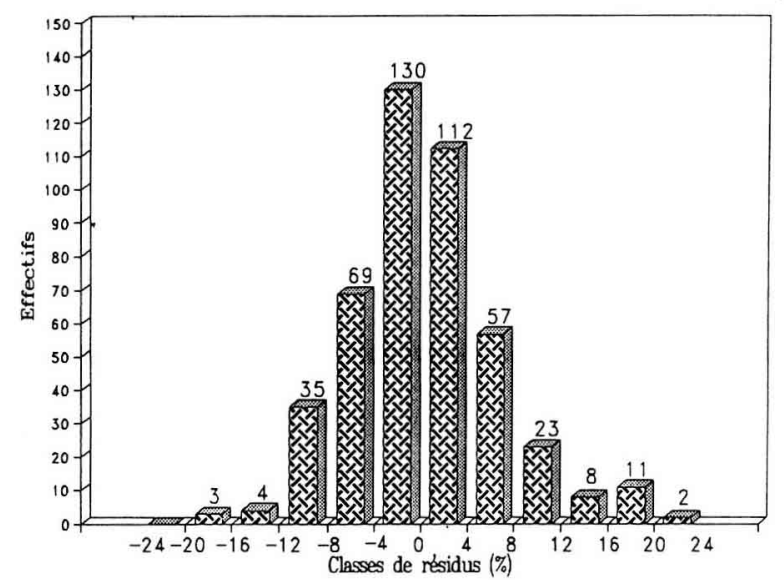

Fig 2. Histogramme des résidus du modèle prédictif (454 observations).

Il est à noter que pour des conditions raisonnables, ce modèle fournit des estimations de pertes de rendement totalement aberrantes. Par exemple, Renan semée le 10 octobre 1990, sur une parcelle à précédent non céréale à paille, située à plus de $40 \mathrm{~km}$ du littoral, a une estimation de perte de rendement égale à $290 \%$ (d'après les sommes de températures calculées sur le poste climatique de Rouen) (pour cette même situation, la perte de rendement estimée, par le modèle qui sera retenu, est égale à $15,4 \%)$ ! Ce modèle ne peut être utilisé dans un but prédictif ; il ne sert qu'à mettre en évidence des effets significatifs.

Pour la prédiction, un modèle simplifié est utilisé : il correspond au modèle sans interaction (tableau IV). Aucune estimation aberrante n'est notée avec ce modèle. Les valeurs de ses paramètres sont fournies dans le tableau $V$. Son coefficient de détermination est égal à $45 \%$. L'effet de la présence de tous les critères est significatif : probabilité associée à la statistique de test égale à 0,06 pour la date de semis, comprise entre 0,0001 et 0,0061 pour tous les autres.

Le graphique donnant les pertes de rendement prédites en fonction des pertes réelles (fig 1) montre que les pertes très faibles (0 à $10 \%$ ) sont surestimées, les fortes (25 à $50 \%$ ) sous-estimées. Les pertes de rendement réelles sont comprises entre 0 et $48 \%$, alors que celles prédites ne vont que de 0 à $32 \%$.

L'histogramme des résidus (fig 2) montre que $81 \%$ des résidus sont compris entre -8 et $+8 \%$ du rendement sans maladie, et seulement 3,5\% des résidus ont une valeur absolue supérieure à $16 \%$.
Tableau V. Modèle prédictif retenu, pour la HauteNormandie et la Picardie.

\begin{tabular}{lr} 
Facteurs et covariables & Paramètr \\
& \\
\hline & \\
Moyenne général & 26,57 \\
Année (de récolte) & \\
1978 & $-4,81$ \\
1979 & $-7,73$ \\
1980 & $-3,05$ \\
1981 & 7,93 \\
1982 & 2,27 \\
1983 & 1,16 \\
1984 & $-6,70$ \\
1985 & 1,20 \\
1986 & $-7,23$ \\
1987 & 5,15 \\
1988 & 0,16 \\
1989 & 6,94 \\
1990 & $-2,67$ \\
1991 & 7,38 \\
& \\
Distance à la mer & \\
0 à 20 km & \\
20 à 40 km & 3,18 \\
< 40 km & $-3,90$ \\
& $-3,78$ \\
Note de résistance à la rouille jaune & $-0,94$ \\
& \\
Note de résistance aux septorioses & $-0,86$ \\
Note de résistance à l'oìdium & $-0,76$ \\
& \\
Nature du précédent & \\
Céréale à paille & \\
Autre & \\
& \\
& \\
&
\end{tabular}

Date de semis

(somme de températures)

0,0051

Ce modèle fournit une estimation de la perte de rendement due aux maladies, pour une parcelle, une combinaison de techniques culturales et un climat donnés. Exemple: Pour une parcelle située à plus de $40 \mathrm{~km}$ du littoral, ayant un précédent betterave, et pour la variété Renan (Note de résistance à la rouille jaune égale à 8 , aux septorioses 4 , et à l'oïdium 6), semée le 10 octobre 1990 (soit $530^{\circ} \mathrm{C}$ pour le poste de Rouen), on évalue la perte de rendement due aux maladies à : $26,57+7,38+(-3,78)+(-1,90)+8 \times(-0,94) 4 \times(-0,86)$ $+6 \times(-0,76)+530 \times(0,0051)=15,4 \%$ du rendement sans maladie.

L'étude des résidus en fonction des covariables et facteurs explicatifs ne révèle aucune relation particulière. La variance résiduelle est due à la variabilité individuelle des observations et aux autres covariables et facteurs importants qui n'ont pu être introduits dans le modèle.

L'erreur quadratique moyenne sur la structure particulière des données est $\mathrm{MSE}=45,1 \%$ au 
carré de la perte de rendement (étant donné que l'unité de la variable que nous étudions est un pourcentage, l'erreur quadratique moyenne est un pourcentage au carré) pour le modèle prédictif (équation [a]), et $53,6 \%$ au carré pour le sousmodèle simple, où les valeurs prédites sont égales aux moyennes annuelles de dégâts observés. L'erreur quadratique moyenne pour la validation croisée, MŜEcv pour le modèle prédictif, est égale à $45,7 \%$ au carré (équation [b]). Ceci ne contredit pas l'aptitude du modèle à prévoir correctement les dégâts de maladies.

\section{DISCUSSION}

L'histogramme des résidus (fig 2) montre que le modèle prédictif explique bien la variabilité des données. Le graphique des pertes de rendement prédites en fonction des pertes réelles (fig 1) indique que le modèle a tendance au recentrage des valeurs extrêmes, caractéristique plutôt rassurante dans une gptique de prédiction. Enfin, les valeurs des MSE montrent que le modèle prédictifs est meilleur que le sous-modèle simple.

Le modèle prédictif montre le rôle déterminant du climat à travers l'année et la distance à la mer (ces facteurs expliquent $80 \%$ de la somme des carrés expliqués par le modèle). Cette importance est cohérente avec les nombreuses études mettant en évidence le rôle des facteurs climatiques sur les contaminations et le développement des maladies (par exemple Schrödter et Ferhrmann, 1971a, 1971b pour le piétin-verse ; Holmes et Colhoun, 1975 pour les septorioses; Coakley et Line, 1981 ; Coakley et al, 1988 pour
Ia rouille jaune ; Gutsche et al, 1986 pour l'oïdium ; Jugnet, 1988 pour la fusariose de l'épi ; Rapilly, 1991).

La plus grande amplitude inter-annuelle de perte de rendement est de 15,7\% (années 1979 et 1981). Les valeurs des "Least squares means" des années (estimations des pertes de rendement obtenues lorsque les covariables sont fixées à leurs valeurs moyennes) permettent de comparer les années entre elles vis-àvis des dégâts (tableau VI). L'ordre des années est globalement cohérent avec celui établi à l'aide des bilans de campagne publiés annuellement (Paris, 1980 ; ITCF, 1980 ; Piquemal et de la Rocque, 1981 ; de la Rocque, 1983 ; Duveauchelle et al, 1984 ; de la Rocque, 1985 à 1992). Les pertes de rendement associées aux années $1980,1981,1983,1987,1988,1989$ et 1991 sont fortes, correspondant, en effet, à de fortes attaques de rouille jaune $(1983,1988$ et 1989 ), de fusariose de l'épi (1987 et 1991), et de septorioses $(1980,1981,1983,1987$ et 1988). À l'inverse, 1979,1984 et 1986 correspondent effectivement à une faible pression parasitaire. 1985 et 1990 sont également bien classées : ce sont des années à développement parasitaire modéré. Pour 1978, la moindre efficacité des fongicides de l'époque est peut-être à l'origine de la sous-estimation des dégâts. En revanche, la forte valeur qui caractérise 1982 est étonnante, car cette année est notée comme n'ayant pas été marquée par un fort parasitisme.

La distance à la mer intervient également : un essai situé à moins de $20 \mathrm{~km}$ de la mer a $7 \%$ de plus de dégâts qu'un essai localisé à plus de

Tableau VI. Situation des années pour les valeurs moyennes des covariables (option Least squares means de SAS) (modèle prédictif, dégâts de I à III), et en fonction des attaques parasitaires d'après les bilans de campagne.

Dégâts de maladies
Attaques parasitaires

\begin{tabular}{|c|c|c|c|c|c|}
\hline $\begin{array}{c}\text { Faibles } \\
\text { attaques } \\
\text { parasitaires }\end{array}$ & $\begin{array}{c}\text { Maladie } \\
\text { dominante: } \\
\text { oïdium et peu } \\
\text { d'autres maladies }\end{array}$ & $\begin{array}{c}\text { Maladie } \\
\text { dominante : } \\
\text { piétin-verse et peu } \\
\text { d'autres maladies }\end{array}$ & $\begin{array}{l}\text { Maladie } \\
\text { dominante: } \\
\text { piétin-verse, } \\
\text { et attaques } \\
\text { moyennes } \\
\text { e septorioses }\end{array}$ & $\begin{array}{c}\text { Fortes attaques } \\
\text { de septorioses } \\
\text { et attaques } \\
\text { moyennes de } \\
\text { de rouille jaune }\end{array}$ & $\begin{array}{c}\text { Fortes attaques } \\
\text { de rouille jaune } \\
\text { ou de fusariose } \\
\text { de l'épi }\end{array}$ \\
\hline
\end{tabular}

de 8 à $14 \%$

$79,84,86$

de 15 à $19 \%$

90
78

85 80

83,88

$\geq 20 \%$
81

$87,89,91$ 
Tableau VII. Différence de dégâts dus aux maladies selon la note de résistance des variétés du catalogue.

\begin{tabular}{lccc} 
Maladie & Rouille jaune & Septoriose & Oídium \\
\hline $\begin{array}{l}\text { Gamme de notes de résistance } \\
\text { du catalogue (réf 1992) } \\
\text { et exemples de variétés }\end{array}$ & 1 (Arfort, Hobbit) & $\begin{array}{c}\text { à } \\
\text { (Blason, Frandoc) } \\
\text { à }\end{array}$ & $\begin{array}{c}\text { (Apollo) } \\
\text { à }\end{array}$ \\
$\begin{array}{l}\text { Différence de dégâts } \\
\text { entre variétés sensibles } \\
\text { et variétés résistantes }\end{array}$ & 9 (Arche, Estica) & 8 (Open, Slejpner)
\end{tabular}

$40 \mathrm{~km}$ du littoral. Ceci est lié à des températures et des humidités plus fortes près du littoral.

La perte de rendement peut également être diminuée de façon notable en privilégiant l'emploi de variétés résistantes à la rouille jaune, aux septorioses et à l'oïdium. Le tableau VII montrent les diminutions de dégâts obtenues en utilisant la variété la plus résistante par rapport à la variété la moins résistante actuellement disponible au catalogue. Ainsi, on estime, grâce au modèle prédictif, que la variété Renan réduit les dégâts de $15 \%$ grâce à ses relativement bonnes capacités de résistance à ces 3 maladies (notes de résistance à la rouille jaune : 8 , aux septorioses: 4, à l'oïdium : 6).

L'ordre de classement de l'importance des notes de résistance aux maladies dans le modèle est : rouille jaune, puis septoriose et oïdium, ce qui est logique au vu des connaissances sur les maladies dans le Nord de la France (Paris, 1980 ; ITCF, 1980 ; Piquemal et de la Rocque, 1981 ; de la Rocque, 1983 ; Duveauchelle et al, 1984 ; de la Rocque, 1985 à 1992). La rouille jaune, pour les années climatiques qui la favorisent, est la maladie qui provoque les dégâts les plus forts dans cette région; les septorioses viennent en seconde position, puis l'oïdium qui provoque de très forts dégâts lorsqu'il atteint les épis.

La faible gamme de variation des notes de résistance au piétin-verse dans nos données explique vraisemblablement l'absence de cette covariable dans le modèle, bien que le piétinverse soit une maladie importante dans cette région.

Enfin, il est important de souligner que les notes de résistance sont insuffisantes à caractériser le comportement des variétés vis-à-vis des pathogènes (Doussinault, communication personnelle) : les notes traduisent des symptômes et, à symptômes équivalents, les variétés ne réagissent pas toutes de la même façon et peuvent conduire à des pertes de rendement différentes. Ces notes restent cependant les seules caractéristiques disponibles puisqu'il n'est pas envisageable de décrire chaque cultivar par son nom : les variétés sont trop nombreuses, et l'actualisation du modèle pour des variétés nouvelles poserait problème.

La gestion adéquate de la succession de culture permet de réduire les dégâts, ainsi que le soulignent Cook et Veseth (1991) et Polley et Thomas (1991). Le modèle simplifié indique que la perte de rendement est supérieure d'environ $4 \%$ avec un précédent «céréale à paille» à ce qu'elle est avec les autres précédents. Comme dans les travaux de Huet (1986a), cet effet est inférieur à celui de la variété.

L'étude des résidus du modèle en fonction de la nature de l'antéprécédent, pour les 108 observations dont l'antéprécédent est connu, montre que la moyenne des résidus des observations avec antéprécédent céréale à paille semble plus forte que celle des résidus des observations avec antéprécédent non céréale à paille (fig 3 ). Un antéprécédent céréale à paille semble donc augmenter la perte de rendement, mais nous n'avons pu quantifier cet effet.

Enfin, il apparaît qu'un choix adéquat de la date de semis permet de limiter les dégâts : le modèle prédictif montre par exemple que passer d'un semis de début octobre (01/10) à un semis de fin novembre (20/11) diminue la perte de rendement de $2,3 \%$ en moyenne.

La quantité d'azote disponible n'a pas été retenue dans le modèle : elle ne joue pas un rôle significatif sur les dégâts dans cette région. Les données disponibles dans les essais n'ont peut-être pas permis d'estimer la quantité d'azote disponible de façon satisfaisante. La quantité d'azote disponible est, de plus, corrélée positivement à la date de semis $(r=0,49)$. 

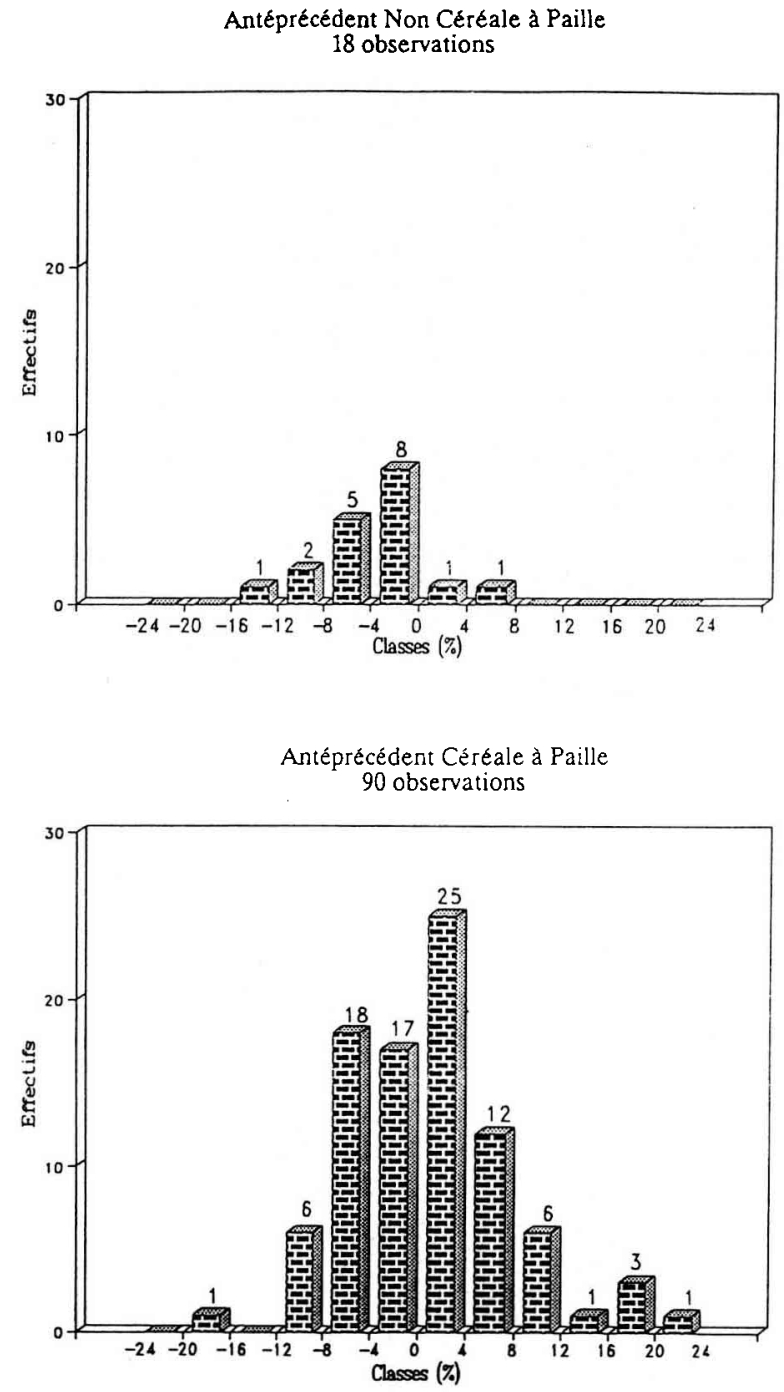

Fig 3. Histogramme des résidus selon l'antéprécédent.

La présence significative de la date de semis dans le modèle pourrait expliquer le fait que l'azote disponible n'apparaisse pas comme covariable significative.

L'analyse des résidus, en fonction de la densité au semis ( 81 observations renseignées) et à la levée (64 observations), ne donne rien de remarquable. Ceci est peut-être dû à la faible gamme de variation de la densité dans les essais.

Un tel modèle ne peut être employé que pour la gamme de ses covariables et de ses facteurs d'entrée ayant servi à la construire. Le domaine de validité du modèle prédictif se limite à des situations à sols de limons, limons argileux, argiles à silex et argilo-calcaires non superficiels ou à des craies, des cranettes, ou des sables moyens à profonds, ayant une quantité d'azote disponible comprise entre 100 et $350 \mathrm{~kg} / \mathrm{ha}$. Les variétés étudiées étaient peu résistances au piétin-verse ; pour des variétés résistantes, la perte de rendement fournie par le modèle devrait vraisemblablement être revue à la baisse.

La structure même du réseau d'essais utilisé pour construire ce modèle introduit un biais dans les estimations des paramètres du modèle. Ainsi, le fait que la structure du réseau ne soit pas la même d'une année sur l'autre induit un biais sur l'effet année : avant 1981, la quantité d'azote disponible est inférieure à $250 \mathrm{~kg} / \mathrm{ha}$ pour plus de $80 \%$ des essais alors que ce pourcentage tombe à moins de 4\% à partir de 1987.

Il est clair que, pour enrichir la modélisation, le dispositif des essais devrait être revu, afin de pouvoir diminuer ce biais et estimer les paramètres des interactions. II faudrait conserver une gamme de variation suffisante pour les différents éléments du système de culture que l'on souhaite étudier, pour des types de sols contrastés et pendant plusieurs années.

\section{CONCLUSION}

L'utilisation du modèle en simulation permet d'envisager la mise au point d'itinéraires techniques à coûts de fongicide réduits. Ainsi, sous une hypothèse de prix du blé à $100 \mathrm{~F} / \mathrm{q}$, on montre (fig 4) que 2 traitements fongicide (nécessaires pour une protection totale contre les maladies et coûtant 7,0 q/ha) sont toujours rentables pour Thésée (variété sensible aux maladies foliaires, très cultivée à la fin des années 1980), semée le 1er octobre, avec un objectif de rendement de $90 \mathrm{q} / \mathrm{ha}$ (pratiques actuellement en vigueur dans la région Haute-Normandie et Picardie). Avec une variété résistante, telle Renan, dans un itinéraire technique plus extensif (rendement objectif de $70 \mathrm{q} / \mathrm{ha}$ ) où l'on retarde le semis de 15 j seulement (semis au 15 octobre), 2 traitements fongicide deviennent alors rentables uniquement 6 années sur 14 . Si le semis a lieu après le 20 novembre, les traitements fongicide sont rentables seulement 4 années sur 14 . Enfin, dans une hypothèse de prix du blé à $75 \mathrm{~F} / \mathrm{q}$, les traitements fongicides sont rentables 13 années sur 14 pour Thésée (semée le $1^{\text {er }}$ octobre), alors que 2 traitements fongicide (coûtant $9,3 \mathrm{q} / \mathrm{ha}$ ) deviennent rentables, pour Renan, seulement 3 années sur 14 pour un semis du 15 octobre, et 1 année sur 14 pour un semis du 20 novembre.

Le modèle permet ainsi à la fois :

- d'identifier, avant expérimentation, les stratégies techniques susceptibles d'être intéressantes dans un nouveau contexte économique ; 
Thessée seméc le $1 / 10$ objectif de rendement $=90 \mathrm{q} / \mathrm{ha}$

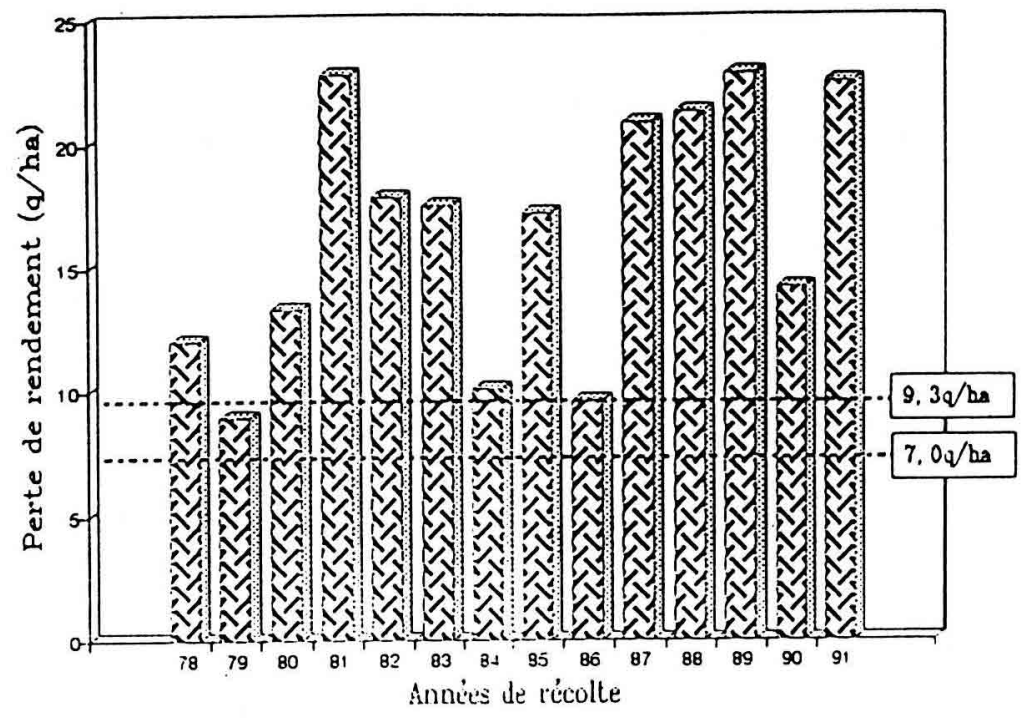

Renan semćc le $15 / 10$ objectif de rendement $=70 \mathrm{c} /$ ha

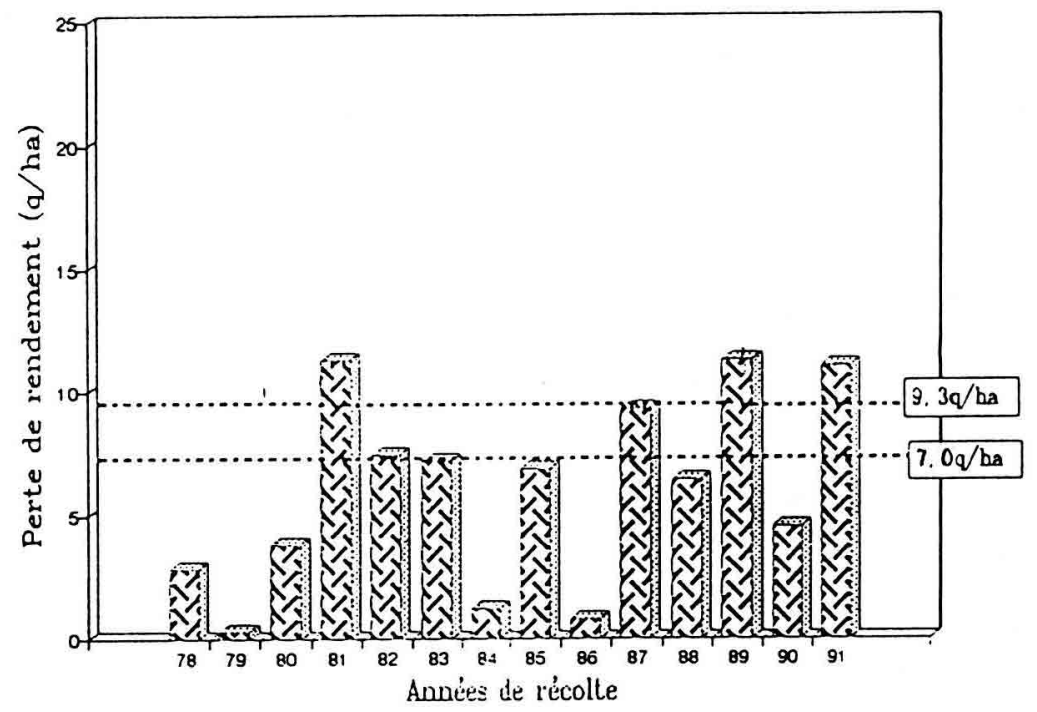

Renan seméc le 20/11

objectif de rendement $=70 \mathrm{q} / \mathrm{ha}$

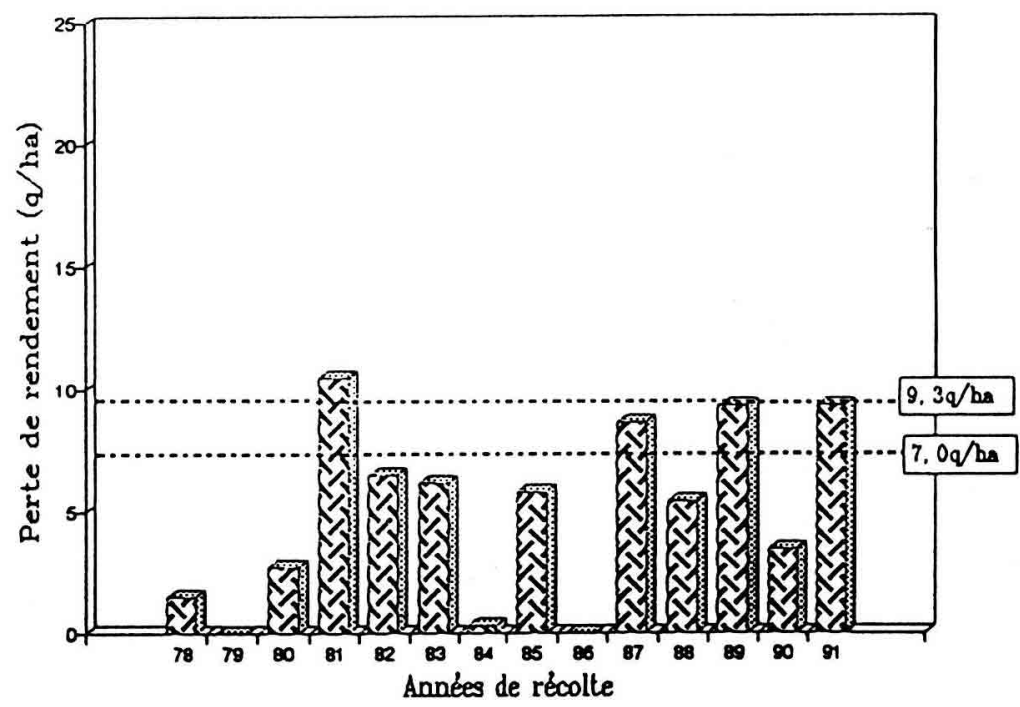

7,0 et $9,3 \mathrm{q} /$ ha correspondent au coût de 2 traitements fongicide pour une hypothèse de prix du blé respectivement a 100 et $75 \mathrm{~F} / \mathrm{q}$

Fig 4. Fonction de distribution, selon les années de récolte, de la perte de rendement calculée par le modèle prédictif. 
- d'intégrer le comportement, sous des climats très contrastés, de variétés qui, telles Renan, viennent seulement d'être sélectionnées.

\section{REMERCIEMENTS}

Nous tenons à remercier l'ITCF et les Chambres d'agriculture des régions Haute-Normandie et Picardie qui nous ont donné accès à leurs bases de données.

Nous exprimons également toute notre reconnaissance à D Wallach (INRA Biométrie, CastanetTolosan) pour ses conseils concernant la validation du modèle, ainsi qu'à C Aubry (INRA-SAD, Grignon) qui nous a guidés lors de l'estimation de l'azote disponible.

Nous remercions enfin les rapporteurs d'agronomie pour le travail important qu'ils ont réalisé pour l'amélioration du manuscript.

\section{RÉFÉRENCES}

Aubry C, Chatelin MH, Poussin JC, Attonaty JM, Massé JM, Meynard JM, Gérard C, Robert D (1992) Déciblé: décision support system for wheat management. In: Poster $4^{e}$ congr inform agric, Versailles, $4 \mathrm{p}$

Bunke O, Droge B (1984) Estimators of the mean squared error of prediction in linear regression. Technometrics 26, 145-155

Caron D (1988) L'oïdium. Perspect Agric 124, 31

Chaboussou F (1985) Santé des cultures. Une révolution agronomique. Flammarion. La maison rustique. Paris, $270 \mathrm{p}$

Coakley SM, Line RF (1981) Climatic variables that control development of strip rust disease on winter wheat. Climatic Changes 3, 303-315

Coakley SM, Line RF, Mc Daniel LR (1988) Predicting stripe rust severity on winter wheat using an improved method for analysing meteorological and rust data. Phytopathology 78 5, 543-549

Cook RJ, Veseth RJ (1991) Wheat health manage. ment. Plant Health Management series. Amer Phytopathol Soc (USA), $152 \mathrm{p}$

Duvauchelle S, Jouanneau R, de la Rocque B (1984) Bilan phytosanitaire de la campagne 1982-1983. Phytoma 356, 10-14

Gutsche V, Müller P, Kluge E, Schultz A (1986) PESTSIM-ERY a model for simulation of infection with powdery mildew (Erysiphe graminis DC). Tag-Ber, Akad Landwirtsch-Wiss DDR, Berlin 242, 5, 101-112

Hébert J (1969) La fumure azotée du blé tendre d'hiver. Bull Tech Inf 244, 755-766

Hollins TW, Scott PR (1980) Epidemiology of eyespot (Pseudocercosporella herpotrichoïdes) on winter wheat, with particular reference to the period of infection. Ann Appl Biol 95, 19-29
Holmes SJl, Colhoun J (1975) Straw-borne inoculum of septoria nodorum and Septoria tritici in relation to incidence of disease on wheat plants. Plant Pathol 24, 63-66

Huet $P(1983 a)$ Influence de la précocité du semis sur le blé tendre. In: Journées d'études ONIC "Rotations Céréalières", Paris, $8 \mathrm{p}$

Huet $P(1983 b)$ Incidence du système de culture et des techniques culturales sur le piétin-verse du blé. Compte rendu de synthèse. Influence des facteurs culturaux sur la progression du piétin-verse chez le blé d'automne. Contrat de programme DGRST, Comité ECAR, Prévisions des risques phytosanitaires, $8 p$

Huet $P$ (1986a) Résultats d'expérimentations spécifiques sur les maladies fongiques d'origine tellurique. In: Les résistances génétiques des cultures céréalières. Colloques INRA, Versailles, 35, 163177

Huet $P$ (1986b) Influence du système de culture sur le piétin-verse du blé. In: Les rotations céréalières intensives. Dix années d'études concertées INRAONIC-ITCF, 1973-1983, INRA, Paris, 95-111

INRA-GEVES (1980) Bulletins des variétés, céréales, GEVES, La Minière, 78280 Guyancourt, $84 \mathrm{p}$

INRA-GEVES (1986) Bulletins des variétés, céréales, GEVES, La Minière, 78280 Guyancourt, $314 p$

INRA-GEVES (1991) Nouvelles variétés de céréales proposées à l'inscription sur la liste $A$ du catalogue français, GEVES La Minière, 78280 Guyancourt, $14 \mathrm{p}$

INRA-GEVES (1992) Nouvelles variétés de céréales proposées à l'inscription sur la liste $A$ du catalogue français, GEVES La Minière, 78280 Guyancourt, $12 \mathrm{p}$

ITCF (1977) Catalogues des variétés. Dépliant, ITCF $\mathrm{Ed}, 3 \mathrm{p}$

ITCF (1980) Catalogues des variétés. Dépliant, ITCF $\mathrm{Ed}, 3 \mathrm{p}$

ITCF (1986) Catalogues des variétés. Dépliant, ITCF $\mathrm{Ed}, 3 \mathrm{p}$

ITCF (1991) Catalogues des variétés. Dépliant, ITCF Ed, $3 \mathrm{p}$

ITCF (1980) Bilan des campagnes 78 et 79 (auteurs non cités). Perspect Agric 35, 49-52

Jugnet MP (1988) La fusariose des épis des céréales : facteurs favorables et effet de l'irrigation. Perspect Agric 124, 179-180

Lechapt G (1983) Les stratégies actuelles de lutte contre les maladies des céréales et les perspectives d'avenir. Brochure Sen Protect Végét, $10 \mathrm{p}$

Linhart H, Zucchini W (1986) Model Selection. John Wiley \& Sons Eds, $301 p$

Meynard JM (1985) Construction d'itinéraires techniques pour la conduite du blé d'hiver. Thèse de Docteur Ingénieur INA-PG, Paris, 258 p + annexes

Meynard JM (1991) Pesticides et itinéraires techniques. Séminaire INRA phytosanitaire, biopesticides, Paris 
Oppitz K, Hoeser K (1978) Ertrags- und Qualitätsbeeinflussung durch N-Spätdüngung und Fungizidbehandlung by Winterweizen. Bayer Landwirtschaft Jahrb 55, 1005-1014

Palti J (1981) Cultural practices and infectious diseases. Adv Ser Agric Sci. Springer Verlag, Berlin, $343 p$

Paris C (1980) Analyse de la campagne céréalière 1978-1979. Perspect Agric 33, 40-45

Piquemal JP, de la Rocque B (1981) Maladies et ravageurs des blés et orges d'hiver : bilan de la campagne 1979-1980. Le réseau de surveillance. Perspect Agric 47, 71-76

Polley RW, Thomas MR (1991) Surveys of diseases of winter wheat in England and Wales, 1976-1988. Ann Appl Biol 119, 1-20

Rapilly F (1982) Agrométéorologie et piétin-verse. Cultivar 149, 14-15

Rapilly $F$ (1991) L'épidémiologie en pathologie végétale. INRA, “Mieux comprendre», $317 p$

Rapilly $F$, Laborie $Y$, Eschenbrenner $P$, Choisnel $E$, Lacroze $F$ (1979) La prévision du piétin-verse sur blé d'hiver. Perspect Agric 23, 30-40

Rémy JC, Hébert J (1977) Le devenir des engrais azotés dans le sol. CR Acad Agric Fr 63 (11), 700-710

de la Rocque $B$ (1983) Bilan phytosanitaire de la campagne 1981-1982. Phytoma 346, 23-25

de la Rocque B (1985) Bilan phytosanitaire 1984 et conseils pour 1985. Phytoma 364, 15-19

de la Rocque B (1986a) Les enseignements de la campagne 1985 en céréales d'hiver. Phytoma 377, 23-26

de la Rocque B (1986b) Importance et répartition géographique des maladies des céréales en France entre 1976 et 1985. Perspect Agric 102, 27-38

de la Rocque B (1987) Après la campagne 1985-1986, faisons le point. Phytoma 387, 10-13

de la Rocque $B$ (1988) Lutte contre les maladies fongiques: quelques enseignements de la campagne 1986-1987. Phytoma 396, 21-28

de la Rocque B (1989) Bilan phytosanitaire 1988. À inquiétudes nouvelles, fongicide nouveaux. Phytoma 409, 22-24 de la Rocque B (1990) Maladies et fongicides : l'année des contrastes. Phytoma 415, 22-30

de la Rocque $B$ (1991) En matière de traitements fongicide, viser juste et frapper fort, est-ce possible ? Phytoma 425, 43-48

de la Rocque B (1992) Campagne 1991. Bis repetita, Fusarioses en plus ! Phytoma 436, 38-40

SAS Institute Inc (1989) SAS/STAT User's Guide, Version 6, Fourth Edition, Volume 2, SAS Institute Inc, Cary, NC, USA, $846 p$

Schrödter H, Fehrmann H (1971a) Ökologische Untersuchungen zur Epidemiologie von Cercosporella herpotrichoides. II. Die Abhängigkeit des Infektionserfolges von einzelmen meteorologischen Faktoren. Phytopathol Z 71, 97-112

Schrödter H, Fehrmann H (1971b) Ökologische untersuchungen zur Epidemiologie von Cercosporella herpotrichoides. III. Die relative Bedeutung der meteorologischen Parameter und die komplexe Wirkung ihrer Konstellationen auf den Infektionserfolg. Phytopathol Z71, 203-222

Schultz $H$, Bodker $L$, Jorgensen $L$ Nistrup, Kirstensen $K$ (1990) Influence of different cultural practices on distribution and incidence of eyespot (Pseudocercosporella herpotrichoïdes) in winter rye and winter wheat. Tidsskr Planteavl 94,221-221

Steinbrenner K, Höflich G (1984) Einfluss acker- und pflanzenbaulicher Massnahmen auf den Befall des Getreides durch Pseudocercosporella herpotrichoïdes (Fron) Deighton und Gaeumannomyces graminis (Sacc) Arx et Olivier Arch. Phytopathol $u$ Pflanzenschutz, bd 20, H6, 469-486

Steinbrenne K, Seidel D (1982) Komplexe Nutzung acker- und pfanzenbaulicher Massnahmen im Rahmen des Pfanzenschutzes. Nachrichtenbl dtsch Pflanzenschutz DDR Berlin 36, 137-140

Stoop P (1992) La PAC va-t-elle modifier les programmes fongicides céréales ? Cultivar $313,26-30$

Wallach D (1991) Models for prediction: evaluation and model selection. Séminaire Modélisaton, Avignon, $58 \mathrm{p}$

Wallach D, Goffinet B (1987) Mean squared error of prediction in models for studying ecological and agronomic systems. Biometrics 43, 561-573 